\title{
Ultrafast All-Optically Controlled 2x2 Crossbar Switch
}

\author{
I. Glesk, K. Il Kang and P.R. Prucnal \\ Princeton University \\ Department of Electrical Engineering \\ Princeton, NJ 08544 \\ Tel: (609) 258-1339; FAX: (609) 258-2158
}

We report the first demonstration of all-optical address recognition at $250 \mathrm{Gbps}$ and selfrouting of photonic packets using all-optically controlled ultrafast $2 \times 2$ switching element. The packet-switching bit-error rate was less than $10^{-9}$. 
Ultrafast All-Optically Controlled 2x2 Crossbar Switch

\author{
I. Glesk, K. Il Kang and P.R. Prucnal \\ Department of Electrical Engineering Princeton University, Princeton, NJ 08544
}

Tel: (609) 258-1339; FAX: (609) 258-2158

For present-day transmission rates in fiber-optic packet-switched networks, routing of the packets is made with opto-electronic $\mathrm{LiNbO}_{3}$ crossbar switches. Switching speed of these devices is practically limited only to several tens of GHz. However in the future optical networks address and data bits might be spaced only picoseconds apart. In these networks new type of ultrafast all-optical devices: a) for address recognition $[1,2]$ and b) for routing are required to eliminate electronic bottlenecks caused by opto-electronic conversion which is necessary to obtain routing control signal for $\mathrm{LiNbO}_{3}$ cross bar switch. One low-power device capable of performing both these functions is the Terahertz Optical Asymmetric Demultiplexer (the TOAD, see figure 1a). We have already demonstrated that for $\Delta \mathrm{x}=\tau \bullet \mathrm{c} / 2$ ( $\mathrm{c}$ is the speed of light and $\tau$ is the bit interval) the TOAD can perform an ultrafast all-optical address recognition when address bits are only 4 ps apart (this corresponds to $250 \mathrm{Gbps}$ bit rate) [1]. If the parameter $\Delta \mathrm{x}=\mathrm{T} \cdot \mathrm{c} / 2$ ( $\mathrm{T}$ is the lengths of the optically compressed photonic packet) the TOAD can be used as an all-optical $2 \times 2$ routing switch.

To demonstrate this, one node of a network of $2 \times 2$ switches was used (see figure 1c). Packets, with 4 ps bit periods, were composed of a large amplitude leading clock pulse, a three bit header, and an empty payload. The switching node consists of an all-optically controlled $2 \times 2$ routing switch (TOAD2) in a switched or unswitched state, an ultrafast routing controller (TOAD1) that sets the state of the routing switch and an optical buffer that matches the delay of the input packet to the processing delay of the routing controller. Before entering the buffer a portion $(10 \%)$ of the packet was split off and sent to the TOAD1 which reads the packet destination address bit. Demultiplexed address bit is then amplified in a Semiconductor Optical Amplifier (SOA) and used as an optical routing control for $2 \times 2$ routing switch (TOAD2). Packets with address bit 2 of value "1" were made to exit output port 2, while packets with address bit of value " 0 " were made to exit output port 1 . Figure $1 \mathrm{~b}$ shows the input multiplexed high intensity clock and two packets: " $1110 \ldots 0$ " and " $1010 \ldots 0$ ". The 4 ps temporal separation of the header bits results in the different headers appearing as cumulative "double height" (101 bit sequence) and "triple height" (111 bit sequence) pulses when observed on bandwidth limitted oscilloscope.

Figures $2 \mathrm{~b}$ and $3 \mathrm{~b}$ are oscilloscope photographs of the output of the routing switch TOAD2 when the clock pulse has been synchronized to demultiplex address bits 2 and 1, respectively. The upper trace shows the output port 1 and the lower trace output port 2 . When bit 1 (always "1"), is used as the address bit, both packets are routed to port 2. This results in zeroes at the output of port 1, and alternate double height and triple height pulses at the output of port 2. However, when bit 2, (alternately a "0" or "1"), is used, the packet with header "111" is always routed to port 2, while the packet with header "101" is always routed to port 1 . This results in triple height pulses at port 2 with zeroes in between, and double height pulses at port 1 with zeroes in between.

We report the first demonstration of all-optical packet switching using all optical routing control where both ultrafast address recognition and routing of photonic packets were all-optically performed on a header in which the bit period is only 4 picosecond in duration (e.g. at $250 \mathrm{Gbps}$ data rate). Packets were self-routed through a node with no need for opto-electronic conversion. 
A new all-optical device, the terahertz optical asymmetric demultiplexer, or TOAD, was used as an optically controlled $2 \times 2$ routing switch and also as an ultrafast all-optical routing controller enable to read individual address bits in this tightly compressed packet header and also set the state of the routing switch . The bit-error rate at the switching element was measured to be less than $10^{-9}$.

References:

[1] I. Glesk, J.P. Sokoloff, P.R. Prucnal, "All-Optical Address Recognition and Self-Routing in a $250 \mathrm{~Gb} / \mathrm{s}$ Packet-Switched Network," Electronics Letters 30, 1322 (1994).

[2] D. Cotter, et. al., "Self-Routing of 100 Gbit/s packets using 6-bit 'keyword' address recognition," Electronics Letters 31, 1475 (1995). 